\title{
Observation of excess quantum noise in a geometrically stable laser
}

\author{
A. M. Lindberg, ${ }^{*}$ M. A. van Eijkelenborg, K. Joosten, G. Nienhuis, and J. P. Woerdman \\ Huygens Laboratory, Leiden University, P.O. Box 9504, 2300 RA Leiden, The Netherlands
}

(Received 30 July 1997)

\begin{abstract}
We have observed excess quantum noise in a geometrically stable He-Xe laser. This was achieved by insertion of an aperture in front of one of the laser mirrors, the aperture diameter being considerably smaller than the beam waist of the lowest-loss Gaussian mode. The measured excess noise is as large as a factor of 15 for the smallest aperture. A simple model is introduced to calculate the transverse excess noise factor using a far-field approximation. We have studied the square and circularly shaped aperture case, both theoretically and experimentally. Our experimental data demonstrate that the overall excess noise factor is given by the product of the transverse and longitudinal excess noise factors, except for the smallest circular apertures, where the results suggest that possible effects of gain guiding and waveguiding need to be considered.
\end{abstract} [S1050-2947(98)01904-0]

PACS number(s): 42.50.Lc, 42.60.Da, 42.55.Lt

\section{INTRODUCTION}

The quantum-limited laser linewidth has been explored both theoretically and experimentally in a variety of situations [1]. Recently there has been an increasing interest in lasers for which the quantum linewidth is enhanced by an excess noise factor, the so-called $K$ factor. This linewidth enhancement arises as a result of the nonorthogonality of the laser-cavity eigenmodes [2-18]. Excess noise is commonly classified as being due to either longitudinal mode nonorthogonality, caused by large localized losses [14-17], or due to a transverse mode nonorthogonality, which occurs in lasers with gain guiding [6,7] and in unstable-cavity lasers $[3,4,8-11]$. Experimentally it has been demonstrated that the transverse excess noise factor can enhance the laser linewidth by more than two orders of magnitude in unstablecavity lasers $[3,8]$ whereas the longitudinal excess noise factor stays much closer to unity; the largest reported value is $7.1[17]$.

Mode nonorthogonality may also occur in a stable cavity by using a sufficiently small aperture. A first theoretical study of the transverse excess noise factor in an apertured stable-cavity laser was very recently presented by Brunel et al. [18]; so far, it has not been observed experimentally. We present here measurements of large excess noise factors in a stable-cavity laser. This is achieved by introducing an aperture in front of one of the laser mirrors, with a diameter that is smaller than the lowest-loss mode diameter. This causes strong diffraction losses and perturbs the transverse eigenmodes in such a way that the true, diffraction-affected eigenmodes become mutually nonorthogonal [19]. This should lead to a transverse excess noise factor $K_{T}$. In addition, a small aperture will also introduce a strong longitudinal inhomogeneity of the laser field, which automatically implies a longitudinal excess noise factor $K_{L}$ [14-17]. Our analysis in Sec. III extends the theory of Brunel et al. [18] by

\footnotetext{
*Present address: Accelerator Laboratory, Department of Physics, Helsinki University, P.O. Box 43, FIN-00014 Helsinki, Finland. Electronic address: Asa.Lindberg@helsinki.fi
}

considering also the longitudinal aspects of the problem. An overall (global) $K$ factor does not in general factorize into a product $K_{L} K_{T}$ in the presence of transverse inhomogeneity [20-22]. For example, in gain-guided semiconductor lasers the longitudinal and transverse field distributions are coupled so no separate excess noise factors $K_{T}$ and $K_{L}$ exist. However, in the special case of a cavity with transversely uniform gain and a single aperture in one of the mirror planes, the transverse factor $K_{T}$ becomes independent of the longitudinal coordinate, leading to a factorization of the global noise factor $K=K_{L} K_{T}$ [22].

The paper is organized as follows. First we present the experimental results in Sec. II. Then we calculate the excess noise factors $K_{T}$ and $K_{L}$ on the basis of a far-field model in Sec. III. In Sec. IV we compare the measurements to the calculations. We end with conclusions in Sec. V.

\section{EXPERIMENTAL RESULTS}

Our He-Xe gas laser operates on the high-gain $\lambda=3.51$ $\mu \mathrm{m}$ transition. The operating pressure is $1.2 \mathrm{kPa}$ and the Xe fraction is about $0.3 \%$. This leads to a full width at half maximum (FWHM) gain bandwidth $\gamma / \pi=232 \mathrm{MHz}$. In a borosilicate gain tube, with a length of $4.5 \mathrm{~cm}$ and an inner diameter of $2.2 \mathrm{~mm}$, an RF discharge is maintained. The cavity, as shown in Fig. 1, consists of one concave gold mirror (radius of curvature $R=60 \mathrm{~cm}$, reflectivity $R_{1}=99 \%$ )

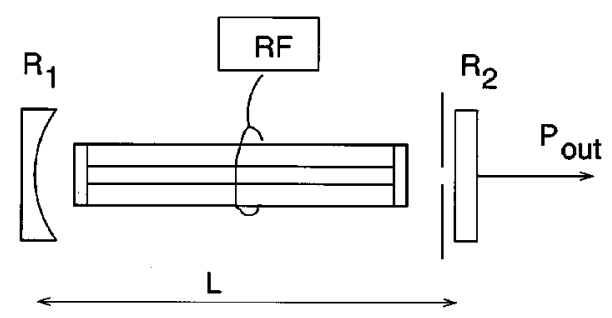

FIG. 1. Schematic drawing of the laser. $R_{1}$ and $R_{2}$ are the mirror reflectivities, RF is the radio-frequency power source, and $L$ the cavity length. The laser output power $P_{\text {out }}$ is coupled out through the flat mirror $R_{2}$. 


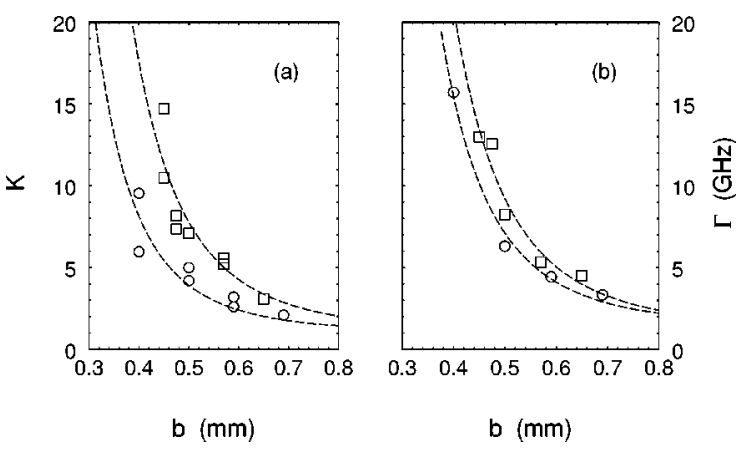

FIG. 2. Measurement results of (a) excess noise factor and (b) cavity loss rate $\Gamma$, both plotted vs $b$, the size of the aperture. The data corresponding to a square (circular) aperture are shown as squares (circles). In (a) the data are fitted to the function $K=1$ $+c_{0} b^{-4}$, and in (b) to $\Gamma=c_{1}+c_{2} b^{-4}$, with $c_{0}, c_{1}$, and $c_{2}$ fit parameters. This $b^{-4}$ dependence follows from our far-field model (see text). The offset parameter $c_{1}$ in (b) accounts for $b$-independent losses, such as the intracavity-window losses and the transmission through the $R=70 \%$ mirror.

and a dielectric flat outcoupling mirror $\left(R_{2}=70 \%\right)$ with a mirror spacing $L=5.4 \mathrm{~cm}$. With no limiting aperture in the cavity we calculate the waist of the unperturbed fundamental Gaussian $\mathrm{TEM}_{00}$ mode to be $2 w_{0}=0.88 \mathrm{~mm}$ on the flat mirror. Right in front of this mirror we insert a square or circularly shaped aperture. The square aperture consists of four razor blades, which can be adjusted to change the size $b$ of the opening (we define $b$ as the edge of the square). The circular apertures consisted of sharp-edged holes with a diameter $b$ between 0.39 and $0.69 \mathrm{~mm}$; the holes were drilled conically from one side into a metallic plate of $1 \mathrm{~mm}$ thickness. The aperture diameters were considerably smaller than the diameter $2 w_{0}$ of the unperturbed Gaussian mode, in order to cause severe diffraction losses. As the aperture size is made smaller, the cavity loss rate increases, which changes the laser linewidth in a standard Schawlow-Townes fashion [1], i.e., in a way not related to the excess noise factor. Therefore, for each aperture that we used, we measured the corresponding cavity loss rate $\Gamma$ using the technique described in [23] to correct for this effect (the correction remains small compared to $K$, i.e., a factor of $1.2-2.7$, since the laser operates in the bad-cavity regime [1]). To measure the quantum-limited linewidth we use the polarizationrotation technique $[8-10,23]$ in which the $\mathrm{He}-\mathrm{Xe}$ discharge tube is placed in a longitudinal magnetic field. The laser linewidth is deduced from the spectral width of the beat frequency between the $\sigma^{+}$and $\sigma^{-}$Zeeman-split laser field components. The linewidth shows the familiar double-valued behavior with output power, in agreement with previous observations $[23,24]$. The measured linewidths can then be compared to the linewidth calculations (see [1]), which yields the excess noise factor. The incomplete-inversion factor $N_{\mathrm{sp}}$ was measured independently from the nonconstant linewidth-power product $\Delta \nu P_{\text {out }}$ as a function of the RF power. An extrapolation to zero RF power gave $N_{\mathrm{sp}}=N_{\mathrm{sp}}^{(0)}$ $+\lambda P_{\mathrm{rf}}$ with $N_{\mathrm{sp}}^{(0)}=1.3(1)$ and $\lambda=0.8(1) W^{-1}$ (see [24]).

The measured excess noise factors, obtained both for square and circular apertures are presented in Fig. 2(a) as a function of the aperture size $b$. Figure 2(a) shows that excess noise can indeed occur in a geometrically stable cavity, with

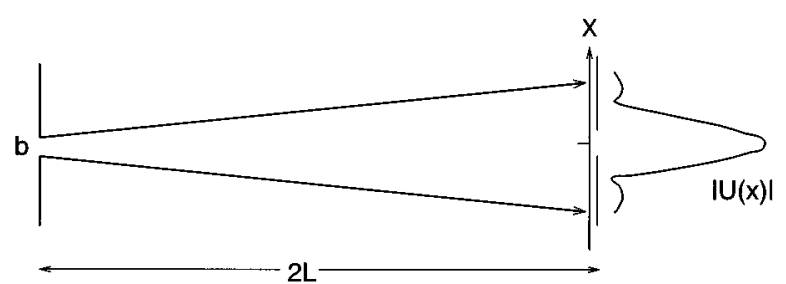

FIG. 3. The circulating transverse mode profile $|U(x)|$ is described as the far-field diffraction pattern at a distance $2 L$ from an aperture with a size $b$.

excess noise factors up to 15 . We find that the magnitude of the excess noise factor is limited by the available gain in the $\mathrm{He}-\mathrm{Xe}$ discharge; when an aperture with a size below 0.39 $\mathrm{mm}$ is inserted in the cavity, the laser cannot be brought above threshold any more. The measurements of the cavity loss rate $\Gamma$ are shown in Fig. 2(b). The dashed curves in both Fig. 2(a) and Fig. 2(b) are fits, which show that both the $K$ factor and the losses contain a $b^{-4}$ dependence as predicted by the far-field model discussed below.

\section{THEORETICAL CONSIDERATIONS}

In order to calculate a transverse excess noise factor $K_{T}$ one needs to know the transverse laser mode intensity profile and wavefront curvature. For very small aperture diameters $b$, we can describe the mode profile after one round trip through the cavity by the far-field diffraction pattern originating from the aperture (see Fig. 3). This approach is valid in the far-field limit, i.e., when $b^{2} \ll 2 L \lambda$. (In the experiments shown in Fig. 2 this condition is not really fulfilled; $2 L \lambda$ $=0.38 \mathrm{~mm}^{2}$ whereas $0.16 \mathrm{~mm}^{2}<b^{2}<0.48 \mathrm{~mm}^{2}$.) In the case of a square aperture we can write for the field amplitude [25]

$$
U(x, y)=U_{0} \frac{\sin \beta_{x}}{\beta_{x}} \frac{\sin \beta_{y}}{\beta_{y}} e^{i k\left(x^{2}+y^{2}\right) / 4 L},
$$

and in the case of a circular aperture

$$
U(r)=U_{0} \frac{2 J_{1}\left(\beta_{r}\right)}{\beta_{r}} e^{i k r^{2} / 4 L} .
$$

The parameter $\beta_{s}=\frac{1}{2} k b \sin \theta_{s}$ with $\tan \theta_{s}=s / 2 L$ and $k$ $=2 \pi / \lambda$. In the case of a square aperture, $s$ stands for the Cartesian coordinates $x$ and $y$, whereas in the case of a circular aperture $s$ is the radial coordinate $r$. For simplicity we have neglected the weak focusing of the $R=60 \mathrm{~cm}$ mirror opposite to the aperture.

To calculate the $K$ factors we choose a reference plane just before the aperture and write the transverse amplitude profile of the wave going to the right as $U$ [see Eqs. (1) and (2)]. The wave traveling to the left at the reference plane has an amplitude given by $V=U$ inside the aperture $(-b / 2<s$ $<b / 2)$ and $V=0$ elsewhere. Using this "cutoff" of the farfield mode profile, the transverse excess noise factor at this reference plane $K_{T}$ can easily be calculated as described in Refs. [2,12]. Assuming a transversely uniform gain the $K_{T}$ factor is independent of the longitudinal coordinate so we are not restricted to this choice of a reference plane; a calculation of $K_{T}$ at different planes along the cavity gives the same result. 


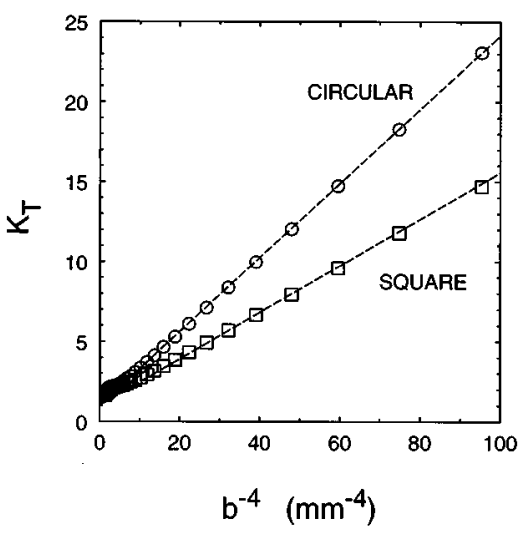

FIG. 4. Transverse excess noise factor $K_{T}$ calculated with the far-field model as a function of $b^{-4}$ for a circular (circles) and a square (squares) aperture. The dashed lines are linear fits to the calculations. For the experimental data shown in Fig. 2(a) the values of $b^{-4}$ lie between 4 and $40 \mathrm{~mm}^{-4}$.

The square and circular data points in Fig. 4 show the calculated $K_{T}$ factors of a square and a circular apertured laser, plotted against $b^{-4}$. Figure 4 clearly shows the $b^{-4}$ proportionality of the $K$ factor. It must be noted that in the case of a variable-reflectivity-mirror (VRM) laser (where the laser mirrors have a Gaussian reflectivity profile) a similar dependence has been found in the small aperture limit [5]. The $b^{-4}$ behavior can be understood as follows. The modulus of the eigenvalue $\alpha$ of the field propagator is obtained by setting $|\alpha|^{2}$ equal to the integral of $|V|^{2}$ divided by the integral of $|U|^{2}$, where the integrals are the two-dimensional space integrals over the (infinite) area of the reference plane (note that $U$ extends in principle to infinity whereas $V \neq 0$ only inside the aperture). In the far-field limit one then finds $|\alpha| \sim b^{2} / 2 \lambda L$. The intensity losses scale as $|\alpha|^{-2}$, i.e., as $b^{-4}$. Using Eqs. (1) and (2) it can easily be shown that in the far-field limit also $K_{T} \propto|\alpha|^{-2}$, and thus $K_{T} \propto b^{-4}$. Apparently, for an apertured stable cavity, there exists a direct relation between $K_{T}$ and the losses. This is in contrast with the $K$ factor in an unstable-cavity laser, for which no obvious relation exists [10]. Another difference between a stable and an unstable cavity is that for the stable cavity $K_{T}$ is larger ( $\sim 1.6$ times) for a circular as compared to a square aperture whereas this is the other way around for the unstable cavity $[9,13]$. Within the far-field model we can ascribe the larger $K_{T}$ value for the circular aperture to the fact that a circular hole of diameter $b$ has less transmission than a square hole with an edge $b$ (when the screen in which the apertures are set is uniformly illuminated). Contradictory to the far-field model we find in the measurements [Fig. 2(a)] that $K_{T}$ is $\sim 2.3$ times smaller (instead of $\sim 1.6$ times larger) for the circular as compared to the square aperture case. This discrepancy will be discussed below.

As a check on the validity of our far-field model we numerically calculated the transverse eigenmodes of the apertured laser using an iterative Fox-Li-type calculation [26]. We find that the $K_{T}$ values calculated this way nicely agree with those calculated with our simple far-field model. Surprisingly, the fact that we have not really fulfilled the farfield condition $b^{2} \gg 2 L \lambda$ does not lead to strong deviations. The advantage of our model, apart from the shorter numeri-

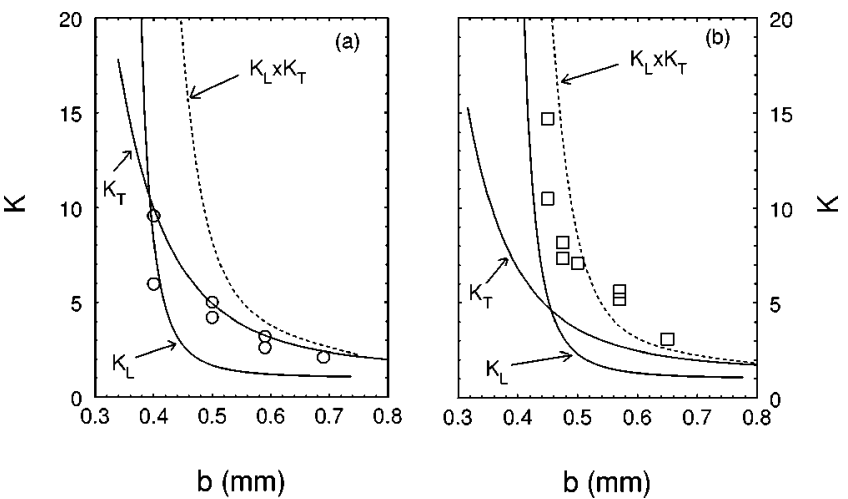

FIG. 5. The calculated longitudinal and transverse excess noise factors (solid curves) plotted together with the measured $K$ factors against the aperture size $b$ of (a) a circular and (b) a square aperture. The product $K_{L} K_{T}$ is shown by the dashed curve.

cal computation times, is that we gain more physical insight.

Apart from the transverse-diffraction effects described above, the apertured laser obviously also has a strong nonuniformity of the longitudinal field distribution. In a onedimensional description the system can be seen as consisting of one highly reflecting mirror $R_{1} \approx 1$ (the gold mirror) and a mirror with effectively a low reflectivity $R_{2}^{\text {eff }}$ (the diffraction loss at the aperture is taken up in the mirror reflectivity $R_{2}$ ). The enhancement of the quantum linewidth in this situation can be calculated from [14-17]

$$
K_{L}=\frac{\left(1-R_{2}^{\text {eff }}\right)^{2}}{R_{2}^{\text {eff }} \ln ^{2} R_{2}^{\text {eff }}} .
$$

According to Eq. (3) $K_{L}$ increases dramatically as the reflectivity $R_{2}$ becomes small. The measured cavity loss rate $\Gamma$ (see Fig. 2) is mainly due to diffraction losses at the aperture in front of the outcoupling mirror. We can, for each value of the aperture size $b$, determine the effective reflectivity of the aperture-mirror combination $R_{2}^{\text {eff }}$ by using the relation $\Gamma=$ $-(c / 2 L) \ln R_{2}^{\text {eff }}$. For the data shown in Fig. 2(a) the corresponding values of the effective reflectivity $R_{2}^{\text {eff }}$ range from $30 \%$ to $0.3 \%$. For simplicity we have neglected the window losses in this calculation (when these are taken into account properly, the calculated values of $K_{L}$ change by only a few percent).

\section{COMPARISON OF THEORY AND EXPERIMENT}

To combine all of the above results we plot in Fig. 5 the calculations of $K_{T}$ and $K_{L}$ as a function of $b$. We have also plotted the experimental results for the circular and square apertures already shown in Fig. 2.

As mentioned above, the measured $K$ factors are smaller for the circular than for the square aperture case, contrary to our far-field model (Fig. 4). In the square aperture case, the product $K_{L} K_{T}$ (dashed curves in Fig. 5) agrees with the data points [Fig. 5 (b)] but not in the circular case [Fig. 5 (a)] [see in particular the smallest aperture points in Fig. 5(a); the measured values lie much below the calculated product of $\left.K_{L} K_{T}\right]$. We think that the discrepancy in the circular aperture case [Fig. 5(a)] can be ascribed to the possible presence of guiding mechanisms such as gain guiding and waveguiding. 
Guiding tends to concentrate the field intensity along the cavity axis, leading to a lower loss, and, thus a to lower $K$ factor. Our discharge tube has a circular cross section. Therefore, due to symmetry reasons, the guiding effects will be more efficient when the mode itself also has a circular symmetry. This hypothesis is corroborated by the fact that the measured losses are $\sim 1.3$ times smaller in the circular case [Fig. 2(b)] as compared to the square case. This is to be compared to the case with no guiding present; the intensity losses scale as $b^{-4}$, i.e., as (area) ${ }^{-2}$, leading to losses being $(4 / \pi)^{2} \sim 1.6$ times larger (instead of $\sim 1.3$ times smaller) in the circular case than in the square case.

We will briefly try to quantify the possible gain guiding and waveguiding mechanisms. The He-Xe discharge has a radial gain distribution, which can lead to gain guiding. In fact, judging from the measurements in [27], gain guiding may already occur when the laser mode is still confined to a region that is considerably smaller than the diameter of the glass discharge tube (the gain coefficient can vary by tens of percent). Waveguiding is to be expected for the smallest aperture cases, where the wings of the far-field pattern after one round trip would be outside the glass tube boundary. We estimate that in the worst case, i.e., the circular $b=0.4 \mathrm{~mm}$ aperture, there is almost $10 \%$ of the power in the beam beyond the glass tube boundary. Due to grazing-incidence reflection, this leads to a reduction of the overall loss.

\section{CONCLUSIONS}

We have shown experimentally that large excess noise factors can arise in stable-cavity lasers. This was achieved by insertion of a small aperture in front of one of the laser mirrors. We have shown in a simple far-field model that the $K$ factor for a stable cavity is directly related to the resonator losses, and that for decreasing aperture size $b$, both rise with a $b^{-4}$ dependence. A large excess noise factor in the case of a geometrically stable laser always implies large losses whereas for an unstable cavity laser large excess noise can be realized already with relatively small losses (e.g., $K_{T} \approx 200$ -300 for $\left.R_{2}^{\text {eff }} \sim 25 \%[3,8]\right)$. In the $K$-factor measurements on a geometrically stable laser with a circular discharge tube we find, in the case of square apertures, agreement with the calculated values of $K_{L} K_{T}$. In the case of circular apertures, our measurements suggest that a simple one-aperture stablecavity model is not sufficient; one needs to consider the possible presence of guiding mechanisms such as gain guiding or waveguiding, which can lower both the losses and the $K$ factor.

\section{ACKNOWLEDGMENTS}

This work was part of the research program of the Stichting for Fundamenteel Onderzoek der Materie (FOM) which was supported by NWO. We also acknowledge support from the European Union under ESPRIT Contract No. 20029 (ACQUIRE) and TMR Contract No. ERB4061PL95-1021 (Microlasers and Cavity QED).
[1] M. P. van Exter, S. J. M. Kuppens, and J. P. Woerdman, Phys. Rev. A 51, 809 (1995), and references therein.

[2] A. E. Siegman, Phys. Rev. A 39, 1253 (1989); 39, 1264 (1989).

[3] Y. J. Cheng, C. G. Fanning, and A. E. Siegman, Phys. Rev. Lett. 77, 627 (1996).

[4] Y. J. Cheng, P. L. Mussche, and A. E. Siegman, IEEE J. Quantum Electron. 30, 1498 (1994).

[5] J. L. Doumont, P. L. Mussche, and A. E. Siegman, IEEE J. Quantum Electron. 25, 1960 (1989).

[6] K. Petermann, IEEE J. Quantum Electron. 15, 566 (1979).

[7] H. A. Haus and S. Kawakami, IEEE J. Quantum Electron. 21, 63 (1985).

[8] M. A. van Eijkelenborg, A. M. Lindberg, M. S. Thijssen, and J. P. Woerdman, Phys. Rev. Lett. 77, 4314 (1996).

[9] M. A. van Eijkelenborg, А. M. Lindberg, M. S. Thijssen, and J. P. Woerdman, Opt. Commun. 137, 303 (1997).

[10] M. A. van Eijkelenborg, А. M. Lindberg, M. S. Thijssen, and J. P. Woerdman, Phys. Rev. A 55, 4556 (1997).

[11] G. Yao, Y. C. Chen, C. M. Harding, S. M. Sherrick, R. J. Dalby, R. G. Waters, and C. Largent, Opt. Lett. 17, 1207 (1992).

[12] G. H. C. New, J. Mod. Opt. 42, 799 (1995).

[13] M. A. Rippin and G. H. C. New, J. Mod. Opt. 43, 993 (1996).

[14] W. A. Hamel and J. P. Woerdman, Phys. Rev. Lett. 64, 1506 (1990).

[15] W. A. Hamel and J. P. Woerdman, Phys. Rev. A 40, 2785 (1989)
[16] M. P. van Exter, W. A. Hamel, and J. P. Woerdman, Phys. Rev. A 43, 6241 (1991).

[17] The value $K_{L}=7.1$ can be deduced from the data in S. J. M. Kuppens, M. P. van Exter, M. van Duin, and J. P. Woerdman, IEEE J. Quantum Electron. 31, 1237 (1995) for the case $R_{1}$ $=100 \%, R_{2}=0.5 \%$, taking into account the outcoupling efficiency $\eta$.

[18] M. Brunel, G. Ropars, A. Le Floch, and F. Bretenaker, Phys. Rev. A 55, 4563 (1997).

[19] M. Brunel, G. Ropars, A. Le Floch, and F. Bretenaker, Phys. Rev. A 55, 781 (1997).

[20] J. Arnaud, Opt. Quantum Electron. 18, 335 (1986).

[21] Y. Champagne and N. McCarthy, IEEE J. Quantum Electron. 28, 128 (1992).

[22] G. Nienhuis and K. Joosten (unpublished).

[23] A. M. Lindberg, M. A. van Eijkelenborg, and J. P. Woerdman, IEEE J. Quantum Electron. 33, 1767 (1997).

[24] S. J. M. Kuppens, M. A. van Eijkelenborg, C. A. Schrama, M. P. van Exter, and J. P. Woerdman, IEEE J. Quantum Electron. 32, 383 (1996).

[25] F. L. Pedrotti and L. S. Pedrotti, Introduction to Optics (Prentice-Hall International, Englewood Cliffs, NJ, 1987).

[26] To perform the calculations, we used the software package PARAXIA developed by J. L. Doumont, A. E. Siegman, and J. A. Ruff, and distributed by SCIOPT Enterprises, 7189 Wooded Lake Dr, SanJose, CA 95120.

[27] P. A. Wolff, N. B. Abraham, and S. R. Smith, IEEE J. Quantum Electron. 13, 400 (1977). 